\title{
Philosophiques
}

\section{La nature de la complémentarité entre le raisonnable et le rationnel chez Rawls}

\section{Geneviève Nootens}

Volume 24, numéro 1, printemps 1997

Avez-vous lu Rawls?

URI : https://id.erudit.org/iderudit/027422ar

DOI : https://doi.org/10.7202/027422ar

Aller au sommaire du numéro

\section{Éditeur(s)}

Société de philosophie du Québec

ISSN

0316-2923 (imprimé)

1492-1391 (numérique)

Découvrir la revue

Citer cet article

Nootens, G. (1997). La nature de la complémentarité entre le raisonnable et le rationnel chez Rawls. Philosophiques, 24(1), 25-41.

https://doi.org/10.7202/027422ar

\section{Résumé de l'article}

Rawls symbolise les niveaux de raisonnement moral public et non public par l'utilisation des notions de raisonnable et de rationnel. Dans le contexte du pluralisme raisonnable qui caractérise les sociétés démocratiques libérales, il propose une articulation de ces notions qui doit permettre aux citoyens d'adhérer à leurs propres conceptions générales du bien sans pour autant compromettre l'existence d'une conception commune de la justice. $\mathrm{R}$ vise à articuler doctrines particulières et conception commune de façon à laisser la place à l'expression des convictions personnelles des individus sans par ailleurs donner une signification égoïste à l'association politique. Cependant, lorsque Rawls précise le contenu de cette conception commune de la justice et la motivation des individus à y adhérer, il menace dangereusement la spécificité morale qu'il accorde en principe à la notion de raisonnable, symbole de cette raison publique, commune, partagée par les citoyens. Après avoir démontré cette difficulté, je souligne en conclusion deux raisons qui y contribuent, soit d'une part la prétention de Rawls de définir un libéralisme strictement politique et d'autre part la difficulté (présente chez la plupart des théoriciens du libéralisme contemporain) d'articuler une conception cohérente et acceptable de la motivation morale.
Ce document est protégé par la loi sur le droit d'auteur. L'utilisation des services d’Érudit (y compris la reproduction) est assujettie à sa politique d'utilisation que vous pouvez consulter en ligne.

https://apropos.erudit.org/fr/usagers/politique-dutilisation/ 


\title{
LA NATURE dE LA COMPLÉMENTARIIÉ ENIRE LE RAISONNABIE ET IE RATIONNEL CHEZ RAWLS*
}

\author{
PAR \\ GeneViève Noo tens
}

RÉSUMÉ : Rawls symbolise les niveaux de raisonnement moral public et non public par l'utilisation des notions de raisonnable et de rationnel. Dans le contexte du pluralisme raisonnable qui caractérise les sociétés démocratiques libérales, il propose une articulation de ces notions qui doit permettre awx citoyens d'adhérer à leurs propres conceptions générales du bien sans pour autant compromettre l'existence d'une conception commune de la justice. $I$ vise à articuler doctrines particulières et conception commune de façon à laisser la place à l'expression des convictions personnelles des individus sans par ailleurs donner une signification égoüste à l'association politique.

Cependant, lorsque Rawls précise le contenu de cette conception commune de la justice et la motivation des individus à $y$ adhérer, il menace dangereusement la spécificité morale qu'il accorde en principe à la notion de raisonnable, symbole de cette raison publique, commune, partagée par les citoyens. Après avoir démontré cette difficulté, je souligne en conclusion deux raisons qui y contribuent, soit d'une part la prétention de Rawls de définir un libéralisme strictement politique et d'autre part la difficulté (présente chez la plupart des théoriciens du libéralisme contemporain) d'articuler une conception cohérente et acceptable de la motivation morale.

ABSTRACT: Rawls uses the notions of the reasonable et the rational to symbolise two levels of moral reasoning: a public (common) one and a non-public one. Reasonable pluralism characterizes liberal democratic societies; in this context, Rawls looks for a specific articulation of public reason and nonpublic reasons (the reasonable and the rational) which could allow citizens to pursue their own conception of the good without compromising the adoption of a common standpoint. Rawls thus wants to link the common point of view of public reason with the comprehensive doctrines citizens defend so as to let them free to build on their own conception, without the political association being reduced to a mere modus vivendi of egoist people.

* Je tiens à remercier le Conseil de recherches en sciences humaines du Canada pour la bourse postdoctorale qui m'a permis de travailler à ce texte. 
However, when Rawls specifies how the content of the common conception is to be defined and how citizens are moved to sustain it, he endangers the moral specificity he wants to grant to the notion of the reasonable as the symbol of the public reason citizens share. Having defended this point, I conclude by insisting upon two reasons which contribute to this confusion in Rawls's work: the definition of a political, rather than general, liberalism, and a flawed conception of moral motivation, characteristic of many contemporary liberals.

Le contexte du pluralisme qui caractérise les sociétés démocratiques libérales fixe le but pratique d'une théorie de la justice telle que la conçoit Rawls. Dans ce cadre en effet, il s'agit de définir une conception de la justice spécifiant les valeurs politiques communes des citoyens, citoyens qui adhèrent par ailleurs à divers cadres moraux généraux. Les citoyens doivent s'entendre sur une conception publique de la justice qui leur permettra d'arbitrer leurs revendications conflictuelles en matière de justice politique, tout en les laissant libres de poursuivre leurs propres conceptions du bien.

La théorie de la justice comme équité, c'est-à-dire cette conception particulière de la justice politique que propose Rawls, compose donc avec deux niveaux de raisonnement moral : un niveau public (les valeurs politiques communes qui font l'objet d'un consensus parmi les citoyens) et un niveau non-public (les différentes doctrines complètes sur lesquelles ne s'entendent pas les citoyens et qui ne peuvent par conséquent pas fournir de bases dentente nécessaires ${ }^{1}$ ). L'utilisation des notions de raisonnable et de rationnel par Rawls symbolise ces deux niveaux de raisonnement moral. Le raisonnable (l'idée des termes équitables de la coopération et la motivation morale liée à cette idée) encadre et limite le rationnel (la poursuite par chacun de ses fins et intérêts particuliers).

Rawls propose une articulation particulière de ces notions, articulation qui distingue explicitement sa théorie de la justice de théories où la coopération dérive strictement de la poursuite des intérêts individuels. Pour Rawls, bien que le raisonnable et le rationnel soient complémentaires en ce que ces idées agissent ensemble pour spécifier les termes équitables de la coopération, le raisonnable ne peut pas ètre dérivé du rationnel. Il désigne un mode spécifique de raisonnement pratique qui ne se réduit pas à l'exercice de la rationalité dans la définition et la poursuite des fins "privées "des individus.

1. Rawls utilise en anglais l'expression comprehensive doctrines. Catherine Audard propose de traduire comprehensive par " compréhensives ", mais ce terme n’a pas en français tout à fait la même signification que le terme anglais. J'utiliserai plutôt l'expression doctrines complètes pour désigner ces " doctrines philosophiques, morales et religieuses personnelles qui englobent de manière plus ou moins systématique et complète les divers aspects de l'existence humaine et, donc, qui dépassent les seules questions politiques, les considerant comme un cas particulier d'une conception plus large. "John Rawls, Justice et démocratie, traduction, introduction. presentations et glossaire de Catherine Audard, Paris, Éditions de Minuit, 1993, p. 359. 
Je veux ici défendre la thèse que l'interdépendance étroite entre le raisonnable et le rationnel qui caractérise la théorie de Rawls permet difficilement de comprendre comment il arrive à spécifier la notion de raisonnable sans la fonder sur les considérations liées à la rationalité individuelle. Ces deux notions apparaissent en effet inextricablement liées, de telle sorte que mème au niveau de la théorie morale, la notion de raisonnable semble dépendre de la rationalité individuelle. Or, l'introduction d'un contenu et d'une motivation étroitement dépendants de la rationalité individuelle menace l'affirmation du raisonnable comme forme distincte de raisonnement pratique. En principe, le raisonnable désigne cette base publique, commune, de justification indépendante des doctrines conflictuelles auxquelles les individus adhèrent dans leur utilisation non-publique de la raison. En introduisant le concept de bien premier pour en définir le contenu et en introduisant une dualité dans la justification morale de l'adhésion à la conception politique de la justice (chacun y adhère pour ses propres raisons et ce sont ces raisons particulières qui déterminent la nature de leur adhésion à la conception politique), Rawls ne peut que difficilement préserver la spécificité morale de la coopération.

Afin de comprendre la nature exacte de la complémentarité entre le raisonnable et le rationnel, je me propose de clarifier le type de raisons qui peuvent motiver les individus à soutenir la conception de la justice que propose Rawls. Pour ce faire, je diviserai ce texte en quatre parties. Dans un premier temps, je spécifierai la signification des notions de raisonnable et de rationnel, en insistant davantage sur celle de raisonnable, plus complexe. Dans un deuxième temps, j'expliquerai brièvement comment Rawls conçoit la complémentarité de ces notions. Dans la troisième partie, je m'attarderai à montrer que la spécification et la justification des principes de justice (ces raisons morales que partagent les citoyens) dépendent essentiellement de considérations liées à la rationalité individuelle. Je soutiendrai, contre Brian Barry, l'hypothèse que l'idée de réciprocité représente une composante essentielle de la théorie de Rawls. Enfin, dans la dernière partie. j'exposerai brièvement en quoi cette interdépendance étroite des notions de raisonnable et de rationnel lie ces deux types de motivation au niveau moral. J'espère ainsi montrer que Rawls ne peut pas distinguer aussi clairement qu'il le prétend le raisonnable du rationnel.

\section{Les notions de raisonnable et de rationnel}

\section{La no tion de rationnel}

Rawls utilise ce terme pour marquer la poursuite par chacun de ses fins individuelles et la conception particulière du bien à laquelle ces fins sont généralement liées. Cette notion désigne donc à la fois la capacité morale qu'a chaque personne de développer, poursuivre et modifier une conception du bien et un certain type de raisonnement pratique qui relève de cette capacité. La notion de principes rationnels s'applique à l'agent individuel qui exerce ses capacités de jugement et de délibération dans la recherche de ses fins et intérêts particuliers. Les fins et intérêts de cette sorte peuvent être intéressés ou désintéressés, mais sont toujours individuels par nature : 
The rational (...) applies to a single, unified agent (either an individual or corporate person) with the powers of judgment and deliberation in seeking ends and interests peculiarly its own The rational applies to how these ends and interests are adopted and affirmed, as well as to how they are given priority. It also applies to the choices of means, in which case it is guided by such familiar principles as : to adopt the most effective means to ends, or to select the more probable altemative, other things equal.

Lagent rationnel ne possède pas cette forme particulière de sensibilité morale qui fonde le désir de coopération équitable et que symbolise l'idée de raisonnable.

\section{La no tion de raisonnable}

Pour sa part, la notion de raisonnable réfère essentiellement à la capacité d'un sens de la justice et aux vertus que Rawls associe à ce pouvoir moral. L'idée de raisonnable désigne à la fois le devoir moral de justifier nos jugements politiques par la raison publique (exigence qui découle du principe libéral de légitimitè) et le désir de coopérer avec les autres sur la base de termes acceptables aux yeux de tous. Etre raisonnable implique avant tout la capacité et la volonté de justifier nos jugements politiques en des termes publiquement acceptables. Ainsi définie, la notion de raisonnable se trouve centrée sur la raison comme capacité d'adaptation sociale, qui permet aux citoyens d'ajuster leurs revendications aux exigences de la vie publique. Le raisonnable se distingue du rationnel d'abord et avant tout grāce à cette capacité ${ }^{3}$.

La notion de raisonnable réfère en fait chez Rawls à une base publique commune de justification à partir de laquelle les citoyens peuvent évaluer les principes de justice et les critères publics de discussion utilisés pour régler les questions de justice politique ${ }^{4}$. Le raisonnable constitue une source de justification des jugements politiques qui doit se distinguer d'une prétention (théorique) à la véritê, tout en définissant un point de vue objectif mais ancré dans la tradition démocratique libérale. Il s'agit donc d'un point de vue intersubjectif mais dépendant de la conception kantienne de la personne, c'est-à-dire de la conception des citoyens comme personnes morales libres et égales. Pour servir de base à la raison publique, la conception de la justice doit être indépendante des doctrines conflictuelles affirmées par les citoyens dans leur utilisation nonpublique de la raison (qui relève de leur capacité morale rationnelle d'avoir une conception du bien). En effet, la justification raisonnable d'une conception de la justice doit partir de ce que nous partageons. La notion de raisonnable définit également le caractère impartial du libéralisme rawlsien. Remarquons dès maintenant (j'y reviendrai plus

2. J. Rawls, Political Liberalism New York, Columbia University Press, 1993 , p. 50. Voir aussi p. 51 : * Every interest is an interest of a self (agent), but not every interest is in benefit to the self that has it. "

3. Ibid., p. 53-54.

4. La notion de raisonnable correspond en fait aux exigences d'une conception de l'objectivité telle que la presente Rawls. Sur ces exigences voir Ibid., p. $110-112,121$. 
loin) que, pour Rawls, cette exigence d'impartialité n'a pas une signification altruiste, mais bien de réciprocitét.

\section{La complémentarité du raisonnable et du rationnel}

Rawls spécifie avec soin que les idées de raisonnable et de rationnel sont indépendantes, au sens où sa théorie ne les dérive pas l'une de l'autre. Rawls veut ainsi se distinguer explicitement des théories, comme celle de D. Gauthier par exemple, qui prétendent dériver le raisonnable du rationnel afin de lui donner un fondement solide. Ce genre de tentative semble lui paraītre contraire à la nature du raisonnable, car de telles tentatives suggèrent que ce type particulier de motivation morale ne possède pas de statut fondamental. Rawls admet par contre qu'il n'est peut-être pas possible de prouver que le raisonnable ne peut pas ètre déduit du rationnel ${ }^{6}$.

Bien que le raisonnable ne puisse pas ètre dérivé du rationnel, ces idées s'avèrent cependant complémentaires dans la conception de la justice que propose Rawls :

[W]ithin the idea of fair cooperation the reasonable and the rational are complementary ideas. Each is an element in this fundamental idea and each connects with its distinctive moral power, respectively. with the capacity for a sense of justice and the capacity for a conception of the good. They work in tandem to specify the idea of fair terms of cooperation, taking into account the kind of social cooperation in question, the nature of the parties and their standing with respect to one another.

As complementary ideas, neither the reasonable nor the rational can stand without the other. Merely reasonable agents would have no ends of their own they wanted to advance by fair cooperation; merely rational agents lack a sense of justice and fail to recognize the independent validity of the claims of others ${ }^{7}$.

L'articulation de ces deux niveaux distincts de l'expérience morale se définit par la priorité du juste sur le bien : les termes équitables de la coopération encadrent la poursuite par chacun de ses fins personnelles. En d'autres mots, le raisonnable (l'idée de réciprocité et de mutualité dans la coopération) présuppose et subordonne le rationnel : il le présuppose, car il exige que les membres du groupe soient motivés par une conception du bien ; il le subordonne, car il limite les fins qui peuvent être poursuivies ${ }^{8}$. Rawls utilise en fait les notions de raisonnable et de rationnel pour marquer la distinction faite par Kant entre les deux formes de raison pratique (pure et empirique), la première s'exprimant dans l'impératif catégorique et

5. Ibid., p. XIX-XX, 16-17, 50, 54. Ceci correspond au fait que dans la position originelle les parties sont dites mutuellement désintéressées. Rawls souligne en effet que le caractère impartial de la justice comme équité dépend essentiellement du fait qu'elle se présente comme raisonnable plutôt que comme vraie.

6. Ibid., p. 51-53. "The best we may be able to do is to show that the serious attempts (Gauthier's is an example) to derive the reasonable from the rational do not succeed, and so far as they appear to succeed they rely at some point on conditions expressing the reasonable itself. "Ibid., p. 53.

7. Ibid., p. 52 .

8. J. Rawls, "Kantian Constructivism in Moral Theory ", The Joumal of Philosophy, vol. 77, $\mathrm{n}^{\circ} 9$, septembre 1980, p. 528-530. 
la deuxième dans l'impératif hypothétique. Le raisonnable constitue un a priori épistémologique (au niveau moral) par rapport au rationnel, en ce sens qu'il établit le cadre moral à l'intérieur duquel les individus peuvent librement poursuivre leurs fins particulières. Dans cette optique, les raisons fournies par cette capacité morale impliquent une forme distincte de raisonnement pratique qui subordonne en principe les autres formes de délibération ${ }^{10}$.

Le raisonnable constitue ainsi le moment où Rawls introduit une conception non-instrumentale de la communauté dans sa théorie, puisque cette notion symbolise ce qu'il y a de spécifiquement moral dans la coopération sociale. En effet, la société politique et les fins qui l'unissent acquièrent une signification morale ne découlant pas strictement de la rationalité individuelle (bien que cette idée fasse également partie intégrante de l'idée de coopération équitable). Rawls distingue la notion de société bien ordonnée ${ }^{11}$ de l'idée de société privée. La société politique ne doit pas ètre unie autour d'une seule doctrine morale, mais les citoyens ne sont pas pour autant dépourvus de fins communes puisqu'ils partagent la même conception politique de la justice et, par conséquent, une fin politique fondamentale. Ces fins communes constituent un bien individuel et social pour les citoyens $^{12}$. Rawls tient par conséquent à distinguer le consensus sur la conception politique de la justice d'un simple modus vivendi: le consensus par recoupement qui exprime l'unité de la société politique possède en effet une valeur morale propre. La conception de la justice est une conception morale, affirmée sur des bases morales ${ }^{13}$.

L'idée de raisonnable implique, comme nous l'avons vu, de justifier en termes publiquement acceptables nos jugements politiques. Ce caractère public symbolise la reconnaissance de la validité indépendante des revendications des autres et limite ainsi ce qui peut légitimement se justifier politiquement (par exemple, il n'est pas

9. J. Rawls, "Themes in Kant's Moral Philosophy * dans Eckart Förster, ed., Kant's Transcendental Deductions. The Three Critiques and the Opus Postumum, Stanford, Stanford University Press, 1989, p. 87-88; Political Liberalism, p. 25 , n. 28 et p. $48-49$, n. 1.

10. Samuel Freeman, "Contractualism, Moral Motivation, and Practical Reason ", The Joumal of Philosophy, vol. 88, $n^{\circ} 6$, juin 1991, p. 297-298. C. M. Korsgaard souligne fort justement que le fait que la raison pratique puisse échouer à motiver ne justifie aucunement le scepticisme par rapport à la raison pratique pure elle-mème. Christine M. Korsgaard, "Skepticism About Practical Reason ". The Journal of Philosophy, vol. 88, n ${ }^{\circ} 1,1986$, p. 5-25. Il suffit de noter que certaines personnes peuvent effectivement être motivées par cette forme de raison pratique qu'est le raisonnable, soit en tant que désir d'ordre plus élevé, comme le soutient Rawls, soit parce qu'elles croient avoir à remplir un devoir categorique sans nécessairement désirer le remplir (cette possibilitẻ est mentionnee par Charles Larmore, Modernité et morale, P.U.F., 1993, p. 40-41).

11. Lexpression "sociêté bien ordonnée * désigne dans la théorie de la justice comme équité un type idéal de société répondant à trois conditions : 1) tous acceptent (et savent que les autres acceptent) les mémes principes de justice ; 2) sa structure de base est publiquement reconnue comme satisfaisant à ces principes ; 3 ) ses citoyens ont effectivement un sens de la justice et se conforment aux institutions de base. Political Liberalism, p. 35.

12. J. Rawls, "The Priority of Right and Ideas of the Good ". Philosophy and Public Affairs, vol. 17, $n^{\circ} 4,1988$, p. 269-270; Political Liberalism, p. 202204.

13. J. Rawls, Political Liberalism, p. 147. 
raisonnable d'utiliser la coercition pour imposer une conception spécifique du bien). La notion de raisonnable exprime ainsi une contrainte substantielle, soit le respect des personnes en tant que " sources autonomes de revendications légitimes", selon l'expression de Rawls. C'est principalement grâce à ces êléments que Rawls montre que le raisonnable ne peut pas être dérivé du rationnel, au sens où l'on aurait besoin de la notion de rationnel pour expliquer la motivation à être raisonnable.

Je vais maintenant examiner deux types de considérations qui me semblent exiger que l'on nuance cette démonstration du caractère " non dérivé " du raisonnable. D'une part (ce que j'examinerai dans la section suivante), le contenu introduit pour définir les principes de justice qui spécifient les raisons que les citoyens partagent et qui fondent leurs relations sociales relève essentiellement de la rationalité individuelle. La possibilité du consensus par recoupement dépend en effet de l'introduction du concept de bien premier, concept qui désigne la structure rationnelle commune des conceptions du bien que poursuivent les citoyens. D'autre part (ce point fera l'objet de la dernière section du texte), au niveau de la théorie morale elle-même, l'indépendance de la notion de raisonnable ne semble pas assurée, à cause d'une dualité dans la justification morale de l'adhésion à la conception politique de la justice.

\section{Raisons de justice et idée de réciprocité}

La dé termination des raisons de justic e

Jusqu'à présent, nous avons examiné la signification de la notion de raisonnable en tant qu'idée. Il faut également déterminer le contenu de cette idée, contenu spécifié par le contenu des termes équitables de la coopération, c'est-à-dire les deux principes de justice $^{14}$. Rawls clarifie les principes de justice appropriès à la façon dont se conçoivent les citoyens d'une société bien ordonnée par le biais du mécanisme de représentation que constitue la position originelle. Il serait inutilement long ici de revenir sur la description complète de la position originelle. Rappelons simplement que la représentation de la conception de la personne sous une certaine forme dans la position originelle permet de spécifier une conception de la justice acceptable pour tous les citoyens en tant que personnes morales libres et égales ${ }^{15}$. Rappelons également que dans la position originelle, les deux capacités des personnes morales lla capacité d'avoir une conception du bien et celle de posséder un sens de la justice) se trouvent représentées de façon formelle: le sens de la justice des parties n'a pas de contenu et les parties ne connaissent pas leurs fins particulières (elles ne connaissent que la structure d'une conception du bien) ${ }^{16}$. Les parties savent qu'elles doivent en arriver à un accord sur une conception publique de la justice, mais uniquement à partir de leur rationalité.

14. Ibid., p. 94.

15. Voir, par exemple, J. Rawls, "Les libertés de base et leur priorité ", Critique, vol. 45, $\mathrm{n}^{\text {os }}$ 505-506, juin-juillet 1989, p. 437-438 : "Kantian Constructivism *, p. 518-519, 522, 530, 570; Political Liberalism, p. 223.

16. J. Rawls, "Kantian Constructivism ", p. 547. 
Or, à ce niveau d'exposition de la théonie, la notion de rationnel, en tant que type de raisonnement pratique et de motivation morale, joue un róle primordial. En effet, les parties dans la position originelle ont pour but de faire avancer le bien précis des personnes qu'elles représentent. Comme ces conceptions du bien leur sont inconnues, elles déterminent ce bien grâce à l'introduction du concept de bien premier. Ce concept s'adresse au problème suivant : étant donnée la diversité des conceptions du bien, comment peut-on arriver à définir une compréhension publique de la justice ? On y arrive, explique Rawls, en supposant une similarité partielle des conceptions du bien permises par les principes de justice, c'est-àdire une idée commune de l'avantage rationnel. Cette similarité réside dans le fait que les citoyens se voient comme motivés par leurs deux capacités morales et que l'avancement de leurs conceptions particulières du bien exige les mêmes biens premiers. Il est rationnel de désirer les biens premiers, car ils constituent les conditions nécessaires pour concevoir et réaliser un projet rationnel de vie. De cette similaritè des citoyens, on déduit les principes de justice. Les biens premiers permettent ainsi de définir une base publique de comparaisons interpersonnelles en matière de justice sociale. Les principes de justice doivent assurer l'accès à ces biens de façon équitable par leur régulation de la structure de base de la société $e^{17}$.

Les parties dans la position originelle ne sont donc motivées que par la réalisation du bien précis des personnes qu'elles représentent. Dans cette optique, elles ne peuvent invoquer de considerations relatives au sens de la justice qu'en tant que moyen du bien d'une personne ${ }^{18}$. Rappelons, par exemple, les raisons de l'adoption des libertés de base et de leur priorité dans la position originelle. Ces raisons relèvent de deux types de considérations, soit les considérations relatives à la capacité d'une conception du bien et les considêrations relatives à la capacité d'un sens de la justice. Relativement à la capacité d'avoir une conception du bien, les raisons d'adopter les libertés de base et leur priorité réfèrent principalement à la liberté de conscience. Celle-ci prime à trois points de vue : parce que le pouvoir moral d'avoir une conception du bien fait partie intégrante de toute conception du bien, parce que ce pouvoir moral constitue un moyen du bien d'une personne et finalement parce que les parties dans la position originelle sont responsables de protéger

17. J. Rawls, "Les libertés de base et leur priorité ", p. 440 ; J. Rawls, "Social Unity and Primary Goods ", dans Amartya Sen et Bernard Williams, eds, Utilitarianism and Beyond, Cambridge University Press, 1982, p. 160-165: "The Prionity of Right ", p. 256-257, 259; "Kantian Constructivism ", p. 525 : Political Liberalism, p. 180 ; J. Rawls, Théorie de la Justice, Paris, Seuil, 1987, p. 473. Les biens premiers comprennent : a) les libertés de base (pensée et conscience, association, liberté et intégrité des personnes, règle de droit, libertés politiques) ; b) la liberté de mouvement et d'occupation; c) les pouvoirs et prérogatives des postes de responsabilité, surtout dans les principales institutions politiques et économiques; d) le revenu et la richesse; e) les bases sociales de l'estime de soi ("Social Unity *, p. 162 ; Political Liberalism, p. 75-76, 178-190). Sur les raisons particulières de l'utilisation de cette liste de biens premiers, voir "Social Unity *, p. 165-166, et "Les libertés de base et leur priorité ", p. 440-441.

18. J. Rawls, Political Liberalism, p. 315-316. 
l'intégrité des conceptions du bien de ceux qu'elles représentent, dans le cadre du pluralisme ${ }^{19}$.

Relativement au sens de la justice, les parties ont trois raisons d'adopter des principes de justice assurant les libertés de base et leur priorité. La première raison est double : un cadre juste et stable de coopération constitue un avantage pour la conception du bien de chacun ; et la conception de la justice spécifiée par les principes de justice s'avère la plus stable, principalement à cause de la priorité des libertés de base. Cette stabilité repose en grande partie sur la congruence entre le juste et le bien permise par les principes de justice :

TThe most stable conception of justice is one that is clear and perspicuous to our reason, congruent and unconditionnally concemed with our good, and rooted not in abnegation but in affurmation of our person. The conclusion argued for is that the two principles of justice answer better to these conditions than the other alternatives precisely because of the basic liberties taken in conjunction with the fair value of the political liberties (...) and the difference principle $e^{20}$.

En second lieu, l'importance fondamentale de l'estime de soi convainc les individus que leur conception du bien vaut la peine d'être poursuivie. L'estime de soi se trouve soutenue à la fois par les libertés de base, qui garantissent le développement et l'exercice des deux pouvoirs moraux, et par la nature publique de cette garantie. Finalement, les parties sont motivées par le fait qu'une socièté démocratique ordonnée par les deux principes de justice représente un bien plus important que le bien d'individus laissés à eux-mêmes ou limités à de petites associations. Des principes offerts aux parties dans la position originelle, les principes de justice proposés par Rawls s'avèrent les plus efficaces pour la réalisation de ce bien, puisqu'ils véhiculent la conception des citoyens considérés comme libres et égaux et qu'ils contiennent une idée de réciprocité appropriée à de telles personnes engagées dans la coopération sociale ${ }^{21}$.

L'adoption des libertés de base et de leur priorité dépend donc dans tous les cas de raisons relatives au bien individuel et déterminé des personnes représentées dans la procédure de construction. Pour Rawls, il ne s'agit pas d'hétéronomie et ce contenu ne constitue pas un impératif hypothétique, car le concept de bien premier exprime les besoins, et non les désirs, des personnes morales. Rawls spécifie que les besoins des citoyens diffèrent des désirs en ce qu'ils sont objectifs : " [...] that is, they express requirements of persons with certain higher-order interests who have a certain role or status. If these requirements are not met, they cannot maintain their role and status, or achieve their essential means ${ }^{22}$. "La motivation des parties dans la position originelle s'avère donc, selon lui, tout à fait appropriée à la représentation des personnes morales ${ }^{23}$. Il faut interpréter en ce sens le postulat du désintérêt mutuel.

19. Ibid., p. 310-314

20. Ibid., p. 317.

21. Ibid., p. 318-322.

22. Ibid., p. 189, n. 20

23. "Once such persons are characterized by the moral powers, it is proper that they should strive to realize and exercise these capacities, and be moved by 
Par ailleurs, il faut bien entendu aujourd'hui considérer la théorie du bien comme rationalité dans l'optique d'une conception politique de la justice, et non d'une doctrine complète. Cependant, la théorie du bien comme rationalité demeure toujours à l'origine de la séquence des idées du bien que comporte la théorie de la justice comme équité. Rawls présente en effet ces idées du bien comme construites selon une séquence qui commence avec le bien comme rationalité $\hat{e}^{24}$. Cette séquence permet de parler du "bénéfice mutuel de la justice mutuelle ", ce qui fonde la justification du consensus par recoupement sur la théorie étroite du bien comme rationalité plutōt que sur le désir de justice des citoyens :

For goodness as rationality allows us to say that things are good (within the political conception) if they have the properties it is rational for us to want as free and equal citizens, given our rational plan of life. From the point of view of the parties in the original position mutual justice meets this condition As citizens in society we normally want justice from everybody else. Much the same holds for the political virtues ${ }^{25}$.

Le désir de coopérer s'avère alors inextricablement lié à l'avantage rationnel qu'ont les citoyens à s'entendre sur des termes équitables de coopération. La signification exacte du raisonnable en tant qu'idée morale provient essentiellement de son caractère public en tant que devoir moral de justification par la raison publique et désir de coopérer sur la base de termes acceptables pour tous. Cependant, on peut légitimement douter que ce caractère public soit suffisant pour préserver l'indépendance du raisonnable par rapport au rationnel. Les contraintes raisonnables encadrant les délibérations de parties dans la position originelle expriment, bien entendu, des considérations substantielles (notamment contre les inégalités dont les individus ne peuvent ètre tenus responsables, la richesse par exemple). Cependant, la façon dont ces contraintes se trouvent intégrées à la conception de la justice s'avère plutôt représentative de la façon dont est construite la position originelle; les contraintes raisonnables se trouvent intégrées essentiellement en tant que limites à ce qui peut ètre poursuivi comme conception du bien, sans nécessairement influencer substantiellement le raisonnement moral individuel.

Au niveau de la construction de la conception de la justice, la théorie véhicule donc effectivement une complémentarité entre le raisonnable et le rationnel, mais uniquement dans la mesure où le consensus par recoupement porte sur une "idée commune de l'avantage rationnel", une "similarité partielle des conceptions du

what I have called their "highest-order" interests. "J. Rawls, "Kantian Constructivism ", p. 547. Les buts des parties ne sont donc pas égoïstes (Ibid., p. 527-531). Mon intention ici n'est pas de relancer le débat sur le fait que le concept de bien premier implique une conceptualisation particulière de ce que signifie " poursuivre une conception du bien ", conceptualisation qui relève d'un idéal spécifique de la personne (voir, par exemple, les critiques initiales de Schwartz et Nagel à la suite de la parution de $A$ Theory of Justice). Je veux simplement attirer l'attention sur le fait que bien que l'optique de la théorie ait été redéfinie pour se limiter à un cadre politique. la théorie du bien comme rationalité joue toujours un rôle primordial.

24. J. Rawls, Political Liberalism, p. 207. Voir également "The Priority of Right ", p. 274, n. 32.

25. Ibid., p. 208. 
bien permises par les principes de justice " ; c'est-à-dire uniquement dans la mesure où le juste se trouve défini conceptuellement par les biens particuliers des individus dans ce qu'ils ont de commun selon une conception libérale générale.

\section{Im par tial ité ou ré ciprocit é}

Cette quasi-subordination apparaìt particulièrement claire quand on considère la signification de l'idée de réciprocité dans la théorie rawlsienne. Rawls précise très clairement que l'impartialité du libèra lisme politique qu'il définit doit être interprétée non pas dans un sens altruiste, mais bien dans l'optique de l'idée de réciprocité. Les termes équitables de la coopération spécifient une idée de réciprocité qui, écrit Rawls, se situe entre l'idée altruiste d'impartialité et l'idée d'avantage mutuel $^{26}$. Le raisonnable ne représente ni un état altruiste, ni un égoïsme :

[The reasonable (with its idea of reciprocity) is not the altruistic (the impartial acting solely for the interests of others) nor is it the concern for self (and moved by its ends and affections alone). In a reasonable society, most simply ilustrated in a society of equals in basic matters, all have their own rational ends they hope to advance, and all stand ready to propose fair terms that others may reasonably be expected to accept, so that all may benefit and improve on what every one can do on their own ${ }^{27}$.

Or, il me semble y avoir ici une distinction fondamentale entre l'impartialité au sens moral où on l'entend habituellement et l'idée de réciprocité sur laquelle Rawls met l'accent. Kymlicka, par exemple, insiste sur le fait que l'impartialité correspond à l'exigence morale de traiter les individus équitablement en reconnaissant leur valeur morale égale. Il décrit ainsi la "règle d'or "que reconnaissent selon lui les cinq théories rivales de la philosophie morale contemporaine :

Adopting the moral point of view (...) requires that we sympathetically attend to people's interests and circumstances, try to understand how things look from their point of view, and give due weight to their well-being. Adopting the moral point of view requires that we "put ourselves in other people's shoes ", and ensure that our actions are acceptable from their point of view as well as our own This, of course, is the basic idea underlying Jesus's "Golden Rule " (do unto others as you would have them do unto youl. This Golden Rule is found not only in Christian ethics, but is also appealed to by deontologists like Kant, utilitarians like Mill, contractarians like Scanlon, and care "theorists like Gilligan $^{28}$.

L'idée de réciprocité a cependant une signification particulière : elle implique l'importance du bénéfice mutuel de la coopération. Comme le reconnait Barry, l'avantage mutuel comme critère de justice

26. Ibid., p. 16-17, 50

27. Ibid., p. 54. Attention : quand Rawls utilise le terme "réciprocité ", il ne veut pas dire que dans sa théorie le statut moral d'un individu dépend de sa capacitè à contribuer à la richesse sociale. Buchanan emploie le terme "justice comme réciprocité " pour désigner ce genre de théorie ; voir Allen Buchanan. "Justice as Reciprocity versus Subject-Centered Justice ". Philosophy and Public Affairs, vol. 19, n 3, été 1990, p. 227-252.

28. Will Kymlicka, "Moral Philosophy and Public Policy: The Case of NRTs ", Bioethics, vol. $7, n^{\circ} 1,1993$, p. 10-11. 
se trouve clairement représenté dans la spécification de la position originelle chez Rawls, qui procède à partir de l'idée que les parties poursuivent leurs propres conceptions du bien sans considération d'équité, bien qu'elles soient soumises à des contraintes raisonnables ${ }^{29}$. Barry suggère d'écarter toute référence à la justice comme réciprocité chez Rawls afin de faire de la théorie rawlsienne une théorie cohérente de la justice comme impartialité :

My reason for bringing up reciprocity here is [...] that I believe it illuminates Rawls's specification of the original position. For the criterion of justice within the theory of justice as reciprocity is mutual advantage over some baseline. And this is, clearly, represented within Rawls's specification of his original position by the provision that the parties are to pursue their own conceptions of the good unconstrained by any considerations of faimess. Of course, the addition of the veil of ignorance means that this pursuit of advartage fails to take off, so that we finish up with a prudential calculation by one person under conditions of radical uncertainty. I suggest, though, that it is the misguided importation of justice as reciprocity that leads Rawls towards the motivational postulate that he puts into the original position ${ }^{30}$.

Selon Barry, une fois écartée cette idée de réciprocité, la justice comme équité représente clairement une théorie de la justice comme impartialité. Ce qui implique, selon les exigences de Barry lui-même, qu'elle réponde à trois caractéristiques. Premièrement, le désir d'agir équitablement doit lui-même motiver les individus (plutôt que la recherche de l'avantage mutuel). Deuxièmement, les règles et institutions doivent être équitables. Enfin, il doit y avoir une correspondance parfaite entre le critère d'équitè et la motivation ${ }^{3 !}$.

La proposition de Barry à l'effet qu'on puisse simplement écarter la motivation rationnelle des parties dans la position originelle pour faire de la justice comme équité une théorie de la justice comme impartialité (au sens où il l'entend) me semble cependant douteuse pour un certain nombre de raisons. Tout d'abord, Rawls opte luimême pour l'idée de réciprocité, qu'il distingue à la fois de l'avantage mutuel et de l'altruisme, pour interpréter la signification de la conception de la justice qu'il propose (il réfère explicitement à Barry en se distinguant de son interprétation) ${ }^{32}$. Ensuite, cette idée de réciprocité lie de façon étroite les deux types de motivation morale, soit l'avantage rationnel de chaque participant et le désir de coopérer. Troisièmement, Barry écarte la question de la stabilité, qu'il considère peu importante une fois déterminés les principes de justice sur la base de la motivation à ètre équitable. Pour Rawls cependant, la question de la stabilité s'avère essentielle, puisqu'il faut gagner le soutien raisonné de citoyens qui partagent certaines valeurs politiques, mais divergent quant aux cadres moraux généraux auxquels ils réfèrent dans leur usage non public de la raison. Finalement, Barry soutient que pour définir une conception impartiale de la justice il faut rechercher une notion de justice indépendante (freestanding) c'est-à-dire "...one that is not subordinated to any one conception of

29. Brian Barry, Justice as Impartiality, Oxford, Clarendon Press, 1995, p. 60.

30. Ibid., p. 60 .

31. Ibid., p. 51 .

32. J. Rawls, Political Liberalism, p. 17, n. 18. 
the good and must therefore call upon a distinctive motive for observing its requirements ${ }^{33}$. "Or, et c'est ce à quoi nous allons nous attarder dans la dernière partie, la structure de la théorie morale chez Rawls ne répond pas à cette exigence.

\section{Conception de la justice et motivation morale}

Rappelons d'abord que dans l'optique d'une définition politique du libéralisme, Rawls postule que les personnes peuvent accepter la conception d'elles-mèmes comme citoyens sans pour autant ètre liées dans d'autres parties de leur vie par les idéaux moraux généraux habituellement associés au libéralisme (l'autonomie et l'individualité par exemple) ${ }^{34}$. Le citoyen affirme deux conceptions, ou deux parties d'une conception, l'une relative à la justice politique (publique) et l'autre dans son utilisation non publique de la raison. Le fait de ne pas imposer notre propre conception générale du bien aux autres citoyens n'implique pas que nous ne puissions nous justifier à nousmémes la conception politique sur la base de notre conception générale. Cela constitue même pour Rawls une condition essentielle de stabilité et de légitimité. La conception publique de la justice se présente donc comme une conception morale, affirmée sur des bases morales, mais par chacun de son propre point de vue. Chacun doit, à partir de son propre cadre moral (en autant qu'il soit raisonnable), se justifier à lui-même la conception politique de la justice et l'articuler avec sa propre conception du bien ${ }^{35}$.

Dans cette optique, le raisonnable joue en quelque sorte le rôle d'une charnière permettant d'articuler différents niveaux de l'expérience morale (politique et non publique). En tant que distinct d'une prétention à la vérité, le caractère raisonnable de la conception politique rend possible l'articulation de celle-ci avec chaque doctrine complète en permettant à chacun d'adhérer au consensus pour ses propres raisons (donc, sans renoncer à sa propre conception générale, pour autant qu'elle soit raisonnable). Rawls postule que nous pouvons converger sur la conception politique de la justice pour différentes raisons morales.

Si on analyse de plus près les raisons que peuvent invoquer les citoyens pour adhérer au consensus par recoupement sur la conception politique de la justice, on remarque que deux cadres distincts peuvent foumir aux citoyens des raisons d'agir les motivant. Il y a, d'une part, cette motivation essentielle liée à l'idéal de citoyenneté, qui relève du cadre politique de la conception publique de la justice. Rawls postule que les citoyens sont fondamentalement motivés par le désir de coopérer entre eux sur la base de termes équitables. L'idéal du citoyen comme libre et égal constitue une source essentielle de motivation, puisqu'il implique que les citoyens désirent réellement réaliser cet idéal. L'idéal du citoyen constitue ainsi ce que Rawls

33. Barry, Justice as Impartiality, p. 76.

34. J. Rawls, "Justice as Fairness : Political not Metaphysical ", Philosophy and Public Affairs, vol. 14, $\mathrm{n}^{\circ} 3$, èté 1985, p. 245.

35. Voir, par exemple. J. Rawls, Political Liberalism p. 38, 134, 140, 143 , 157, 169-171. Ce dualisme origine de la nature spéciale de la culture publique démocratique, marquée par le pluralisme raisonnable (Ibid., p. XXI). 
appelle un conception-dependent desire, ce qui signifie que les individus doivent être prêts à agir sur la base de principes relevant de cette conception ${ }^{36}$.

Mais outre la motivation d'être raisonnable, les individus possèdent également leurs propres raisons pour soutenir le consensus, raisons qui proviennent des doctrines complètes auxquelles ils adhèrent. Dans l'optique de Rawls, la stabilité dépend de la capacité de la conception politique de s'enraciner dans (ou de coexister avec) une variété de doctrines générales raisonnables ${ }^{37}$. Or, cet argument implique une exigence très contraignante pour la théorie. Rawls soutient en effet que les bases sur lesquelles les individus affirment la conception politique détermine la nature de leur affirmation :

All those who affirm the political conception start from within their own comprehensive view and draw on the religious, philosophical and moral grounds it provides. The fact that people affirm the same political conception on those grounds does not make their affirming it any less religious, philosophical or moral, as the case may be, since the grounds sincerely held determine the nature of their affurmation ${ }^{38}$.

Une double considération morale motive donc les citoyens. D'une part, Rawls postule que les citoyens sont fondamentalement motivés par le désir de coopérer entre eux sur la base de termes équitables. Mais le désir d'agir de façon conforme à l'idéal de citoyenneté ne constitue pas le seul désir relatif à une conception (conceptiondependent desire) qui motive les citoyens d'une société bien ordonnée. Si ces individus adhèrent réellement, comme le suppose Rawls, à des cadres moraux généraux auxquels s'intègre d'une façon ou d'une autre la conception politique de la justice, on peut légitimement supposer que ces cadres particuliers sont également générateurs de motivation de cet ordre. Le problème suivant se pose alors : selon l'argument de Rawls, les citoyens effectuent l'articulation entre la conception politique et leur propre doctrine complète sur la base de cette dernière, qui fournit ainsi les raisons morales d'adhérer au consensus sur la conception politique. Les citoyens sont donc effectivement motivés par le désir de coopérer, mais en tant que ce

36. Ibid., p. 49-50, n. 2, p. 81-86.

37. Voir, par exemple, Ibid., p. 143.

38. Ibid., p. 147-148. Voir aussi p. 127 : "In appealing to reasons based on the political conception, citizens are appealing not only to what is publicly seen to be reasonable, but also to what all see as the correct moral reasons from within their own comprehensive view. "Cela pose d'importants problèmes à mon avis, notamment parce qu'il y a alors une concurrence entre les autorités normatives (les sources des jugements de valeur) et parce que cela implique que le lien entre la conception politique et la doctrine générale d'un individu varie avec la nature de son affirmation de la conception politique : ceci constitue une menace à la stabilité, car cela implique que les raisons de soutenir le consensus pèsent de façon différente dans les diverses doctrines. Voir Geneviève Nootens, "Theorie morale et stabilité chez Rawls : le rôle conceptuel de la notion de raisonnable ", présentè à l'ACSP en juin 1995. Noter ici la différence avec l'argument de Kymlicka. Rawls soutient que les individus justifient la conception politique à partir de leur propre cadre et que cela définit la nature de leur affirmation. Kymlicka, par contre, croit que les individus ajustent leurs conceptions particulières pour les rendre cohërentes avec les principes moraux publics. Voir aussi à ce sujet Wayne Norman. - Methodological Rawlsianism ", à paraître. 
désir prend place à l'intérieur de leurs doctrines particulières et que la conception politique peut ètre justifiée sur la base de raisons appartenant à ces cadres particuliers. On peut alors à juste titre se demander si le raisonnable en tant que catégorie morale particulière, censée subordonner les autres formes de délibération, fournit réellement des raisons morales spécifiques.

L'influence des considérations liées à la rationalité individuelle dépasse largement le niveau des motifs pragmatiques ayant trait à la stabilité de la théorie. Les lecteurs de Rawls rencontrent ici un problème de théorie morale. En effet, les principes qui définissent les termes équitables de coopération, et par conséquent les raisons que partagent les citoyens et qui servent à arbitrer les problèmes de justice politique, se trouvent principalement déterminés par l'introduction de considérations rationnelles. Ces considérations rationnelles expliquent également de quelle façon la motivation morale permet d'assurer la stabilité. L'idée de coopération sociale lie ainsi inextricablement les notions de raisonnable et de rationnel, tel que le démontre la signification de l'idée de réciprocité. Enfin, l'affirmation de Rawls que chacun adhère à la conception politique pour ses propres raisons tend à subordonner la motivation relevant de l'idéal de citoyenneté aux raisons relevant des doctrines complètes divergentes auxquelles adhèrent les individus dans l'exercice de leur rationalité individuelle.

On pourrait évaluer qu'il s'agit là de la meilleure façon de justifier une conception de la justice dans le contexte du pluralisme. Cependant, cela ne répond pas à l'exigence théorique que pose Rawls lui-même en ce qui concerne la théorie morale propre qui caractérise sa théorie de la justice. La façon dont la théorie exprime la complémentarité entre les deux niveaux de raisonnement moral rend pour le moins douteuse l'affirmation de Rawls que le raisonnable n'est pas dérivé du rationnel. Bien entendu, il serait pour le moins simpliste de faire de la justice comme équité une théorie du même genre que celle de Gauthier, par exemple. Je crois avoir amplement souligné l'importance de la signification particulière de l'idée de raisonnable chez Rawls. Cependant, il apparait loin d'ètre évident que cette motivation morale spécifique foumisse réellement aux citoyens des raisons d'agir à partir d'un cadre moral indépendant.

La volonté bien affirmée de Rawls de limiter son libéralisme à un cadre politique pour éviter de faire de la justice comme équité une doctrine complète (et par conséquent, controversée) me semble en bonne partie responsable de cette quasi-subordination du raisonnable. En définissant un libéralisme politique, Rawls doit ménager un espace pour une double motivation morale, espace où coexistent le raisonnable et le rationnel. Ainsi, les individus sont motivés à la fois par leur désir de réaliser l'idéal de citoyenneté et par les désirs relatifs à leurs conceptions particulières du bien. La particularité de l'argument de Rawls réside dans le fait que selon cet argument, l'adhésion à la conception politique se fait sur la base de raisons appartenant à ces conceptions divergentes; il devient difficile de comprendre en quoi le raisonnable a véritablement prioritè. La réussite de l'argument de Rawls exige que le désir de réaliser l'idéal de citoyennetê ait prépondérance ; mais l'enracinement de la conception 
politique dans les doctrines particulières peut également signifier la subordination de cet idéal aux raisons particulières, non publiques, que les citoyens peuvent avoir d'adhérer au consensus par recoupement. D'autant plus que la relation entre des bases morales concurrentes (d'une part, le constructivisme qui permet de spécifier la conception de la justice appropriée et, d'autre part, les sources normatives propres à chaque doctrine complète) n'est en fait pas très claire dans la théorie.

Est-ce prendre Rawls en défaut ? Il admet lui-mēme qu'il n'est peut-être pas possible de prouver que le raisonnable ne peut pas ètre déduit du rationnel ; que le mieux que nous puissions faire est peutètre de montrer que les tentatives de déduire le raisonnable du rationnel (celle de Gauthier par exemple) ne peuvent réussir que dans la mesure où elles dépendent de conditions exprimant le raisonnable lui-même ${ }^{39}$. En fait, Rawls se heurte ici à un problème fondamental de la théorie libérale contemporaine : l'indétermination de la nature de la motivation morale. Il reconnait que la motivation à être raisonnable doit prédominer ; mais dans le cadre du pluralisme raisonnable, il croit devoir faire dêpendre cette motivation des conceptions particulières du bien auxquelles adhèrent les individus.

Je ne crois pas qu'il faille écarter complètement cet aspect, qui participe en fait de la reconnaissance de la complexité de la vie morale des individus. Cependant, la théorie libérale actuelle est de peu d'utilité pour démontrer comment la priorité de la justice peut prendre racine dans la structure motivationnelle des individus concrets. Rawls postule que les valeurs politiques dominent les autres valeurs (non publiques). Mais comme le souligne Daniel Weinstock, il n'est pas clair quant à la façon dont nous devons comprendre cette prédominance : s'agit-il d'un argument conceptuel faisant des valeurs politiques un impératif catégorique ou d'une hypothèse empirique suivant laquelle les individus réalisent qu'ils sont plus à même de poursuivre leurs projets dans le cadre des institutions libérales ${ }^{40}$ ?

Si nous pouvons espérer que les citoyens reconnaissent le rôle régulateur des principes de justice (notamment grâce à l'éducation civique), nous ne pouvons postuler que les individus se conformeront aux principes de justice simplement parce que ceux-ci définissent l'idéal politique du citoyen. Sans compter que comme l'ont montré les travaux de Kohlberg, les individus se situent à différents niveaux de développement moral. $\dot{A}$ cet égard, toute tentative postulant la suffisance d'une simple motivation à ètre raisonnable est tout aussi insatisfaisante que l'enracinement de la notion de raisonnable dans les motivations individuelles. Par contre, les débats actuels accordent de plus en plus de place à l'idée de raison publique, et c'est peutêtre effectivement de ce cōté qu'il nous faudra rechercher la force motivationnelle suffisante pour assurer la réalisation des principes de justice. On peut en effet concevoir la raison publique comme une médiation entre, d'une part, les principes de justice et l'idéal dont ils

39. J. Rawls, Political Liberalism p. 53. Cité note 6 ci-haut.

40. Daniel M. Weinstock, "The Justification of Political Liberalism ", Pacific Philosophical Quaterly, vol. 25, 1994, p. 179-180. 
découlent et, d'autre part, la motivation réelle des individus concrets $^{41}$.

Au bout du compte, probablement nous faut-il abandonner l'idée kantienne d'une indépendance absolue du raisonnable. Peut-ètre devons-nous refaire différemment le chemin vers la comprëhension des sources et de la signification de notre vie commune: puisque nous ne pouvons nous résoudre à accepter une loi morale hétéronome et que nous ne pouvons pas non plus poser l'indépendance du raisonnable, ne devrions-nous pas d'abord, comme l'a suggéré Charles Larmore, admettre que la raison pratique n'est pas la source de la morale, puisque la raison ne peut argumenter qu'à l'intérieur d'une tradition morale? Si telle était la leçon que nous devions tirer de la tentative, essentiellement individualiste, de Rawls d'articuler le raisonnable et le rationnel, nous pourrions enfin recentrer l'analyse du libéralisme et mettre un terme à deux décennies de débat sur l'articulation rawlsienne du juste et du bien, du raisonnable et du rationnel.

\section{Université d'Ottawa}

41. Voir à cet effet Geneviève Nootens, "Liberal restrictions on public arguments : Can nationalist claims be moral reasons, in liberal public discourse ?", à paraitre, Canadian Joumal of Philosophy, vol. supplêmentaire, 1997. 